\title{
Acute stroke secondary to AICD lead malposition in the left ventricle
}

\begin{abstract}
Inadvertent lead malposition in the left ventricle is one of the rare complications of implantable cardio-defibrillator (ICD) placement. Unintentional LV lead placement through erroneous cannulation of the subclavian artery is extremely rare. We present the case of an 84-year-old man who underwent elective placement of ICD. Due to excessive bleeding from the ICD pocket, there was concern of arterial puncture, however urgent subclavian angiogram did not reveal any injury to the artery and patient was admitted for observation. The next day patient developed embolic stroke involving multiple territories and further workup with CTA demonstrated ICD lead to be located in left subclavian artery extending into the aorta and into the left ventricle of the heart. He was started on anticoagulation with heparin drip and the malpositioned lead was extracted, and underwent a new implantation successfully. Inadvertent LV lead placement via the subclavian artery is exceedingly rare. Arterial thromboembolic event is the major complication associated with lead malposition in the left ventricle. If the LV lead malposition diagnosed early, the removal of lead is recommended, preferentially while the patient being anticoagulated to reduce the risk of potential thromboembolic complication. Our case demonstrates the serious neurological consequences of inadvertent left heart lead placement and highlights the importance of a high index of suspicion to diagnose early and appropriate management is necessary to avoid future complication.
\end{abstract}

Keywords: implantable cardio-defibrillator complication, stroke, malposition lead, left ventricular lead
Volume 12 Issue $3-2019$

\author{
Ahmad Nawid Latifi, Uzochukwu lbe, \\ Nasheena Jiwa \\ Department of Internal Medicine, St Mary's Hospital, USA
}

Correspondence: Ahmad Nawid Latifi MD, Department of Internal Medicine, St Mary's Hospital, 56 Franklin St,Waterbury, CT 06704, USA, Tel 203-709-3800,

Email nawidlatifi@gmail.com

Received: April 29, 2019 | Published: June 25, 2019

\section{Learning objectives}

Recognize an exceedingly rare occurrence of erroneous LV lead placement during ICD implantation. Recognize the serious adverse event of arterial embolic stroke as a consequence of LV lead malposition. A high index of suspicion is important in order to implement rapid diagnostic and therapeutic management of anticoagulation and lead extraction in the case of a malposition LV lead.

\section{Case presentation}

An 84-year-old gentleman presented to the hospital for a scheduled automatic implantable cardioverter-defibrillator (AICD) implantation. His medical history was significant for coronary artery disease status post coronary artery bypass graft complicated by post-op atrial fibrillation with subsequent ligation of the left atrial appendage, ischemic cardiomyopathy with a left ventricular (LV) ejection fraction of $25-30 \%$ with the prior refusal of AICD placement, mitral regurgitation, and obstructive sleep apnea on continuous positive airway pressure (CPAP), hypertension and IgA deficiency. Prior to this presentation, he had been experiencing recurrent dizzy spells where a 48-hour Holter monitor revealed a predominantly sinus rhythm with intermittent 1 st and 2nd degree AV block as well as complete heart block with a 2.51-second pause. He was also noted to have multiple premature ventricular contractions (PVC) and non-sustained runs of ventricular tachycardia. Besides the dizziness, he was rather asymptomatic and was able to undertake a fair workload without chest pain or shortness of breath. His medications include Aspirin $81 \mathrm{mg}$, atorvastatin $40 \mathrm{mg}$ daily, furosemide $40 \mathrm{mg}$ daily, metoprolol tartrate $25 \mathrm{mg}$ twice daily and spironolactone $25 \mathrm{mg}$ daily. He does not use illicit drugs, smoke cigarettes or drink alcohol. During the ICD implantation, the electrophysiologist was unable to place an atrial lead and after the procedure, the patient was noted to have bleeding from the ICD pocket. There was concern for a potential injury to the left subclavian artery, therefore, vascular surgery was notified urgently. The ICD was already in place and the pressure was being held. There was no obvious hematoma but there was ecchymosis around the area. An urgent subclavian artery angiogram was performed which was significant for a widely patent left subclavian artery without evidence of pseudoaneurysm, active extravasation or any other kind of injury. The left vertebral and internal mammary arteries were visualized and patent. Hemostasis was achieved and the bleeding was attributed to a likely subcutaneous arterial bleed during the time of cut down.

The next morning, the patient was noted to have a change in his neurological exam. He developed dysarthria and ataxia. A stat CT of the head was ordered and showed a new hypodensity within the left cerebellum and chronic periventricular small vessel ischemic changes. An MRI of the brain showed areas of acute infarction in the left parietal lobe, left occipital lobe and left cerebellum with small areas of hemorrhagic transformation in the left parietal lobe infarct. A subsequent MRA of the neck with Intravenous contrast showed a hypointensity in the visualized left subclavian artery which could represent a contrast mixing artifact or thrombus. The stroke was thought to be embolic in nature given the multiple territories involved. Given the MRA findings, a stat CTA of the neck and chest was done for further evaluation. The imaging studies showed the left ICD lead located in the left subclavian artery extending into the aorta and into the left ventricle of the heart. The patient was started on a heparin 
drip with the plan being to retrieve the ICD lead intraoperatively. Under general anesthesia, the patient underwent a left brachial artery cut down with subsequent removal of the defibrillator generator and lead retraction into the subclavian artery and placing a sheath into the artery. An angiogram was performed which showed that the subclavian artery was patent, and the left internal mammary artery (LIMA) and vertebral arteries were visualized indicating there was no evidence of active extravasation or pseudoaneurysm. In addition, there was no evidence of an arteriovenous (AV) fistula. A new AICD was placed and a subsequent angiogram obtained showed no bleeding. The artery was then repaired with good Doppler signal afterward. The patient was hemodynamically stable throughout the procedure and a post-procedure chest X-ray showed a single lead AICD overlying the right ventricle. The patient's neurological status improved and the patient was subsequently discharged 2 days later to a rehab facility (Figure 1-3).

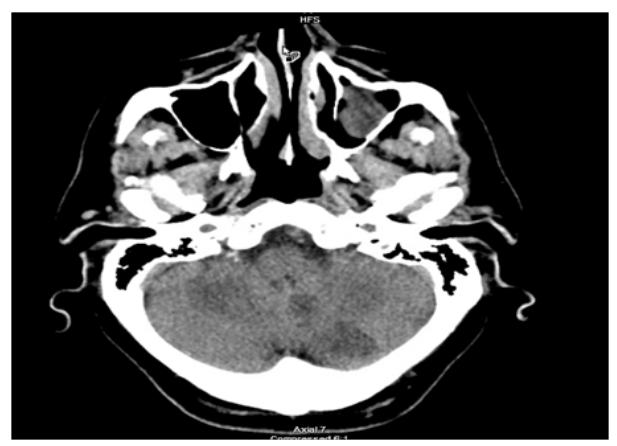

Figure I CT of the head showing hypodensity within the left cerebellum.

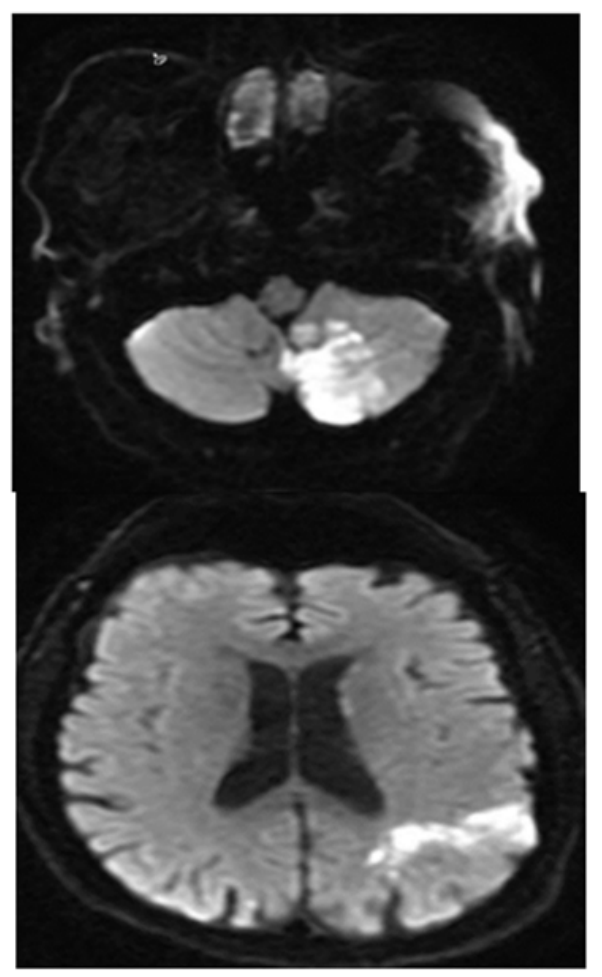

Figure 2 MRI of the brain showing left Cerebellar and left occipital lesions.

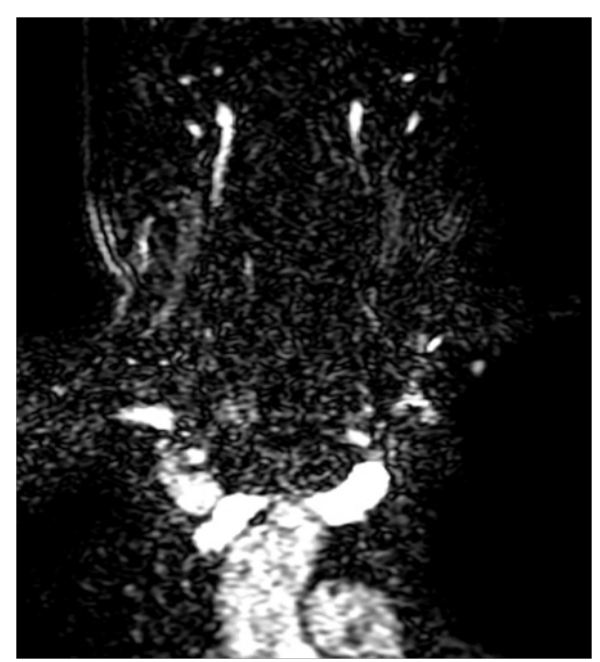

Figure 3 CTA of the neck showing a linear filling defect within the aorta.

\section{Discussion}

As the mortality of cardiovascular disease has declined significantly over the past few decades, more people are living longer with more significant cardiac disease requiring permanent pacemakers (PPMs) and implantable cardioverter-defibrillators (ICDs). It is estimated that more than 100,000 ICD and PPM implantations are performed in the USA annually. ${ }^{1}$ Many modern devices combine pacemaker and ICD in one unit for patients who need both functions. A systematic review of ICD implantation in almost 6800 patients with ischemic heart disease and dilated cardiomyopathy enrolled in randomized control trials, found an overall complication rate of 9.1, including lead displacement $3.1 \%$, pneumothorax $1.1 \%$, hematoma $1.2 \%$ and infection $1.5 \% .{ }^{2}$ Risk factors for lead malpositioning include abnormal thoracic anatomy, underlying congenital heart disease, and operator inexperience. ${ }^{3}$ Inadvertent left ventricle pacing due to malpositioned lead is a known but a rare complication of ICD and pacemaker implantation. The most common reported cause is the passage of the lead through an atrial septal defect (ASD) or patent foramen ovale (PFO). It has also been reported in the setting of ventricular septal and apical perforation. ${ }^{4-6}$ Unintentional LV lead placement through erroneous cannulation of the subclavian artery is extremely rare. The major complication due to lead implantation in LV is systemic thromboembolic events. Van Gelder $^{7}$ reported the rate of thromboembolic (TE) complication in the setting of LV lead malposition causing neurological symptoms at $37 \% .^{7}$ Neurological symptoms range from amaurosis fugax to aphasia and hemiplegia. TE events can occur from 6 months to $61 / 2$ years after implantation of the lead. ${ }^{7}$ The cause of thromboembolism is thought to be from thrombus formation and fibrosis on or around the site of lead implantation. ${ }^{4}$ To the best of our knowledge, this is the first case of cardioembolic stroke, which occurred within 24 hours of inadvertent left ventricle ICD lead placement via subclavian artery. The most likely source of emboli being thrombus formation in the left ventricle in presence of ICD lead.

Although our patient had a history of postoperative paroxysmal atrial fibrillation 3 years ago, he had a left atrial appendage ligation done and a recent Holter monitor and cardiac monitor during hospitalization that did not show any evidence of atrial fibrillation. Other possible complications related to lead malposition in the LV via 
subclavian artery include mitral and aortic valve damage, endocarditis, peripheral arterial thrombosis, diaphragmatic pacing, and loss of capture. ${ }^{7-9}$ Unintentional arterial cannulation can be identified immediately by the color and pulsatile nature of arterial flow and/ or excessive bleeding during or after the procedure. In our patient, excessive bleeding and inability to place atrial lead raised suspicion for subclavian artery cannulation, however subclavian angiography did not reveal any evidence of bleed or pseudoaneurysm and it was difficult to judge exactly where the wires were as the subclavian vein lies directly on top of the artery. In these instances, CT angiography will be the most useful study to accurately diagnose the position of the lead as shown in our case. If misplacement of the lead in the left ventricle is missed initially, EKG and CXR will provide clues to the location of the lead. A right bundle branch block pattern on a 12-lead ECG may be the first indication of LV lead malposition. Although this ECG pattern has good sensitivity, it has low specificity for LV lead malposition since a similar pattern may be seen in the setting of RV dilatation, septal pacing or coronary sinus pacing. ${ }^{4,10} \mathrm{CXR}$ is a useful imaging study to detect the correct position of the lead. For a correctly placed lead into the right atrium and ventricle, a lateral chest X-ray shows the anterior position of the lead and above the diaphragm. On the other hand, a lead located relatively high above the diaphragm in the posterior-anterior view and more posteriorly placed relative to the sternum in the lateral view should suggest misplacement or malposition into the left ventricle or coronary Sinus. ${ }^{11}$ Transthoracic or transesophageal echocardiography can be used to locate the lead

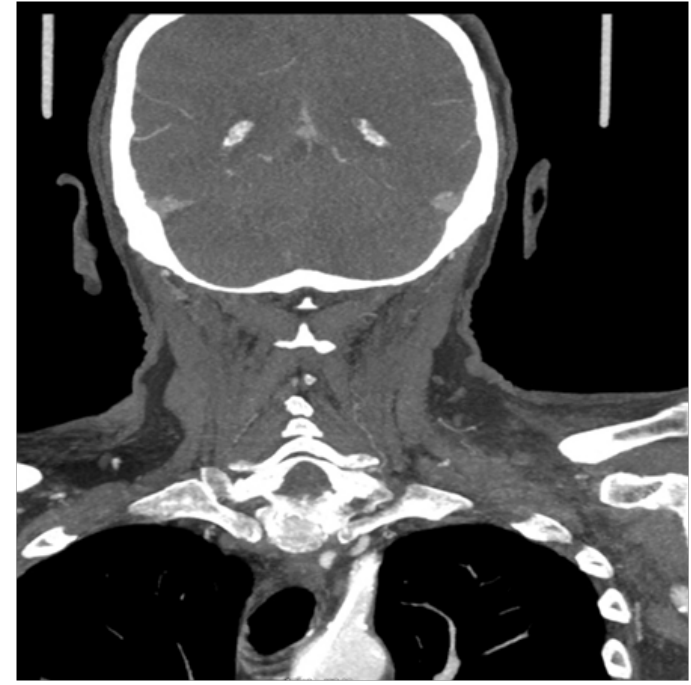

Figure 4 CTA of the neck with IV contrast showing the AICD lead within the aorta.

\section{Acknowledgments}

None.

\section{Conflicts of interest}

Authors declare that there is no conflict of interest.

\section{References}

1. Bollinger BC, Heidenreich J. Current Diagnosis \& Treatment Emergency 6. Stone CK, Humphries RL, editors. New York: Lange Medical Books/ McGraw-Hill; 2008. From cardiac arrhythmias; p. 578-607.

2. Ezzat VA, Lee V, Ahsan S, et al. A systematic review of ICD complications in randomised controlled trials versus registries: is our 'real-world' data an underestimation? Open Heart. 2015;2(1):e000198. accurately and additionally, it will help to differentiate the pathway which the lead has ended up in LV i.e. via an ASD, PFO, or perforation of the interventricular septum. ${ }^{3}$ Chest Computed tomography (CT) is another valuable test which can establish the correct position of the lead and also helps to diagnose lead perforation when echocardiographic findings are equivocal., ${ }^{3,12}$

Management of inadvertent left ventricle lead placement is not well defined. If diagnosed immediately, it is recommended to remove the lead to avoid future thromboembolic complication. Serious complications with percutaneous lead extraction can happen including fatal hemorrhage, pseudoaneurysm formation, arterial dissection or occlusion, and distal embolism. ${ }^{13}$ Sheaths that are advanced over the lead may dislodge thrombi and cause systemic emboli. ${ }^{7}$ Our patient was on heparin drip during the procedure and fortunately, the lead extraction was uneventful. Treatment of malpositioned lead in the left heart diagnosed late includes anticoagulant therapy or lead removal. Lead extraction can be considered and is most appropriate if cardiac surgery has to be performed for other indications. If the malpositioned lead in the left ventricle is not extracted, then lifelong anticoagulant therapy needs to be commenced. Warfarin seems to be effective in primary and secondary prevention of thromboembolic events as antiplatelet therapy as thromboembolism prophylaxis is not recommended. ${ }^{7}$ The role of direct oral anticoagulants (DOAD) in these patients is not known (Figure 4 \& Figure 5).

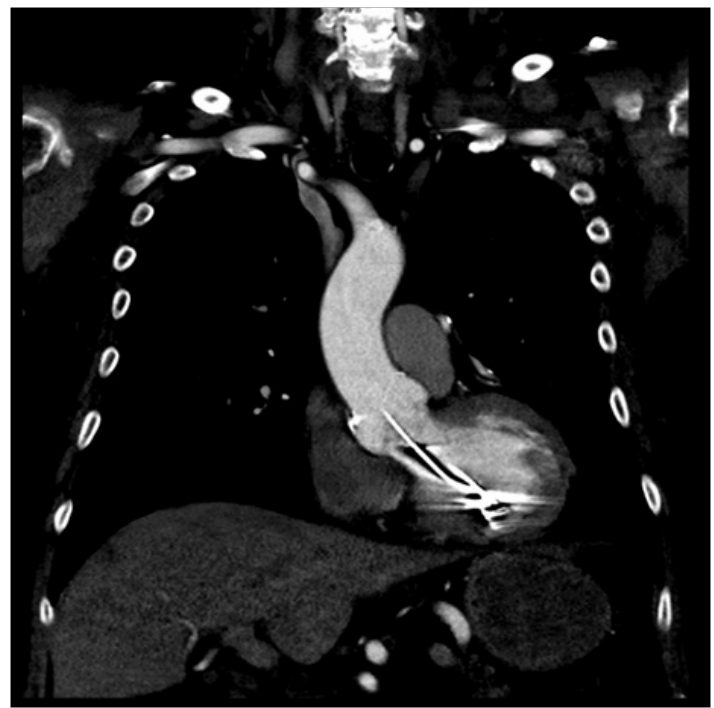

Figure 5 CT of the chest with IV contrast showing the AICD lead within the left ventricle.

3. Trohman RG, Sharma PS. Detecting and managing device leads inadvertently placed in the left ventricle. Cleve Clin J Med. 2018;85(1):6975 .

4. McManus DD, Mattei ML, Rose K, et al. Inadvertent lead placement in the left ventricle: a case report and brief review. Indian Pacing Electrophysiol J. 2009;9(4):224-228

5. Engstrom A. Inadvertent malposition of a transvenous pacing lead in the left ventricle. Herzschrittmacherther Elektrophysiol. 2006;17(1):221-224.

6. Lee WL, Kong CW, Chu LS, et al. Transvenous permanent left ventricular pacing. A case Report. Angiology. 1995;46(3):259-264.

7. Van Gelder BM, Bracke FA, Oto A, et al. Diagnosis and management of inadvertently placed pacing and ICD leads in the left ventricle: a multicenter experience and review of the literature. Pacing Clin. Electrophysiol. 2000;23(5):877-883. 
8. Konings TC, Koolbergen DR, Bouma BJ, et al. Iatrogenic perforation of the posterior mitral valve leaflet: a rare complication of pacemaker lead placement. Journal of the American Society of Echocardiography. 2008;21(5):512.e5-512.

9. Jagadeesh Kumar Kalavakunta, Vishal Gupta, Basil Paulus, et al. An Unusual Cause of Transient Ischemic Attack in a Patient with Pacemaker. Case Reports in Cardiology. 2014. ID 265759, 3 p.

10. Yang YN. Safe right bundle branch block pattern during permanent righ ventricular pacing. J Electrocardiol. 2003;36(1):67-71.
11. Almomani A, Abualsuod A, Paydak H, et al. chronic lead malposition diagnosis and management: discussion of two cases and literature review. Clin Case Rep. 2007;5(3):270-276.

12. Flicker S, Eldredge WJ, Naidech HJ, et al. Computed tomographic localization of malposition of pacing electrodes: the value of cardiovascular computed tomography. Pacing Clin Electrophysiol. 1985;8(4):589-599.

13. Chen X, Bai J, Han F, et al. Percutaneous closure of the inadvertent subclavian artery cannulation during pacemaker implantation. Cardiol J. 2017;24(4):452-454 